\title{
Temporal Variability and the Bulk Lorentz Factor of GRBs
}

\author{
E. Sonbas ${ }^{* 1}$, G. A. MacLachlan ${ }^{2}$, K.S. Dhuga ${ }^{2}{\text {, P. } \text { Veres }^{2} \text {, A. Shenoy }}^{2}$ \\ ${ }^{1}$ Adiyaman University, Department of Physics, 02040 Adiyaman, Turkey \\ ${ }^{2}$ Department of Physics, The George Washington University, Washington, DC 20052, USA \\ E-mail: edasonbas@yahoo.com
}

For a sample of Swift and Fermi GRBs, we have investigated the time variations in the light curves by calculating their minimum time scales (MTS's) using a wavelet method. We show that the MTS for the prompt emission is related to the bulk Lorentz factor in a complex manner: For small $\Gamma$ 's, the variability timescale exhibits a shallow (plateau) region. For large $\Gamma$ 's, the variability timescale declines steeply as a function of $\Gamma$. Evidence is also presented for an intriguing correlation between the peak times, $t_{p}$, of the afterglow emission and the prompt emission variability timescale. These correlations, if substantiated by more precise data, can serve as predictors of unknown Lorentz factors for GRBs.

Swift: 10 Years of Discovery,

2-5 December 2014

La Sapienza University, Rome, Italy

\footnotetext{
${ }^{*}$ Speaker.
} 


\section{Introduction}

The variability of GRB lightcurves and the bulk Lorentz factors are key parameters to our understanding of the production and the propagation of the relativistic outflow that emerges as a powerful GRB jet from the central engine. A measure of the temporal spikiness of the lightcurve potentially provides a quantitative means of probing the regional size of the emission location involved. The Lorentz factor provides a handle on the different energy regimes of GRB evolution i.e., the prompt emission and the afterglow stages.

For a sample of Swift GRBs, we generated mask-weighted, background subtracted light curves by using batgrbproduct, batmaskwtevt, and batbinevt tasks. These lightcurves were generated with a time binning of $200 \mu \mathrm{s}$ in the four standard Swift/BAT energy bands, i.e. $15-25 \mathrm{keV}, 25$ - $50 \mathrm{keV}, 50-100 \mathrm{keV}, 100-150 \mathrm{keV}$. An example of a lightcurve, extracted with a time binning of $64 \mathrm{~ms}$ is shown in Figure 1. For the Fermi sample we extracted light curves for the GBM NaI detectors over the entire energy range ( $8 \mathrm{keV}$ to $1 \mathrm{MeV}$ ). Typically, the brightest three $\mathrm{NaI}$ detectors were chosen for the extraction. Lightcurves for both long and short GRBs were extracted at a time binning of $200 \mu \mathrm{s}$.

Following the work of [6], we used a technique based on wavelets to extract a minimum variability time scale (MTS) for the prompt emission. The wavelet technique, based on determining the logscale diagram, a measure of the log of the variance of the wavelet coefficients versus the frequency in octaves that characterize the lightcurve, provides a robust method for extracting a time scale that allows the separation between frequencies representing white noise (background) and the red noise (GRB signal) (see Figure 1). In practical terms, the MTS is defined as the intersection of the white noise component and the red noise component(see Figure 1).

The majority of the bulk Lorentz factors were taken from [5], and [2]. These authors used several methods for extracting these factors, including the observed peak times for the optical emission. We discarded a number of GRBs from the [5] sample because only a limit was given for the Lorentz factors. Finally, we included a number of Fermi GRBs that have been recently analyzed by [3] and [4].

\section{Results and Discussion}

We explore the possibility of a connection between the time variability and the bulk Lorentz factor by plotting MTS versus the reported Lorentz factor, $\Gamma$ (see Figure 2). The MTS is corrected for the $1 /(1+\mathrm{z})$ time dilation factor, where $\mathrm{z}$ is the redshift for a given burst. The plot exhibits a very interesting knee-like feature in the MTS- $\Gamma$ plane: The MTS shows a shallow (plateau) region for small $\Gamma$ 's and then decreases rather sharply for high $\Gamma$ 's. The mean of the fitted break for the two data sets is $\Gamma=225 \pm 30$. Beyond this $\Gamma$, the MTS follows a steep anti-correlation with $\Gamma$.

In Figure 3, we plot MTS vs. isotropic luminosity (Liso). For most of the bursts, we use the luminosities reported by [5]. Essentially, the MTS-Luminosity plot exhibits the same features seen 


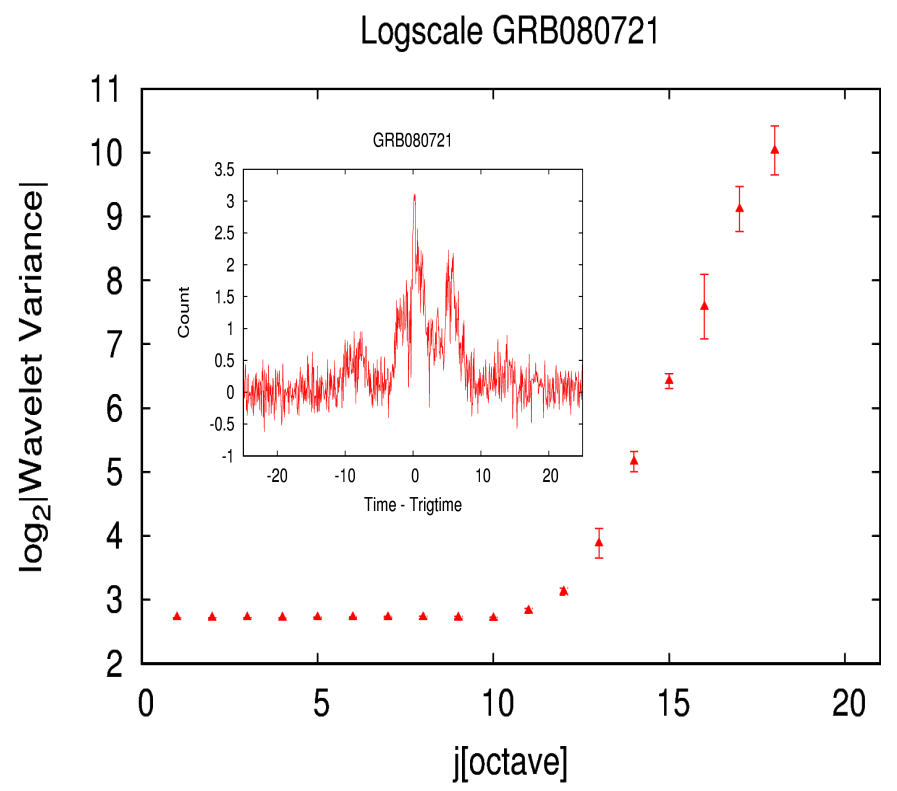

Figure 1: (outer) Logscale diagram for GRB080721: Log(Variance) of signal as a function of octave (inverse frequency). Plateau region is white noise and the sloped region is red noise: Intersection is MTS. (inner) Lightcurve of GRB080721 (64 ms binning)

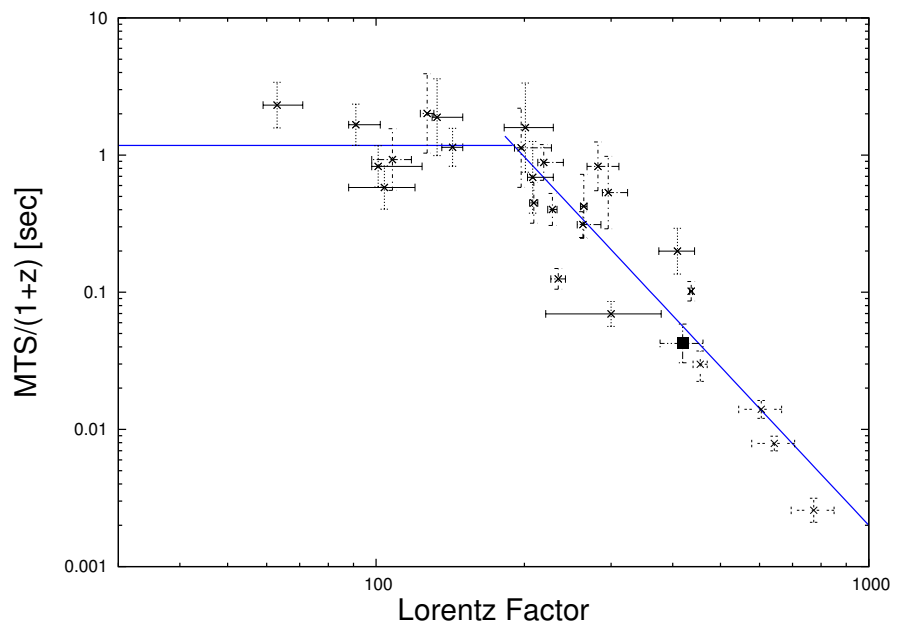

Figure 2: MTS for the prompt emission vs Lorentz Factor. 
in the MTS- $\Gamma$ correlation.

In order to obtain a measure of the sensitivity of the extracted MTS to possible observational bias, we performed a number of simulations. The GRB lightcurves were denoised with a hard-threshold wavelet process described by [1] in which the wavelet coefficients smaller than some threshold are discarded. The remaining coefficients were then used to reconstruct denoised versions of the lightcurves. These simulated lightcurves were then treated as probability distribution functions (PDFs) to seed an event generator sampling from a Poisson distribution. The portion of the PDFs corresponding to the background level was held constant while the amplitude of the signal portion of the PDFs was scaled by various factors $25 \%, 50 \%, 100 \%$, and $200 \%$ relative to the background level. We generated 1000 realizations of lightcurves at each of the above scale factors and the resulting lightcurves were then processed as 'real' GRBs for which an MTS extraction was carried out using the identical wavelet technique employed for the GRBs in the original (observed) sample. The extracted MTS shows some sensitivity to the S/N of the GRB lightcurve as displayed in Figure 4. The effect of this sensitivity, about 1 octave over a variation of a factor of about 5 in $\mathrm{S} / \mathrm{N}$, is reflected in the MTS-Luminosity plot (Figure 3; blue points).

In figure 5, we show the spectral lag vs the bulk Lorentz factor. This plot too shows the same fea-

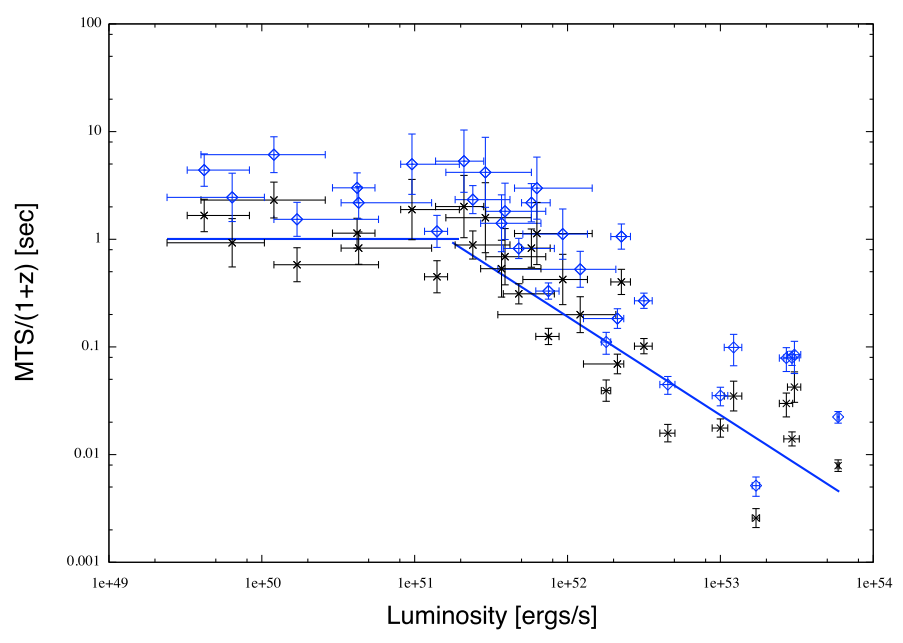

Figure 3: MTS for the prompt emission vs. isotropic Luminosity. Blue points are for simulated light curves.

tures as noted for the MTS-Luminosity correlation. The lag, a temporal difference between arrival times of high-energy and low-energy photons, should be less sensitive to the $\mathrm{S} / \mathrm{N}$ and is therefore less likely to suffer from observational bias as compared to the MTS. Finally, we observe (see figure 6) an interesting connection between MTS and $t_{p}$, the peak times for the afterglow emission.

\section{Summary}

We summarize our main findings as follows:

- The MTS- $\Gamma$ plot exhibits a knee-like feature with a break around $\Gamma=225 \pm 30$. Beyond the break, the MTS decreases steeply with $\Gamma$. 


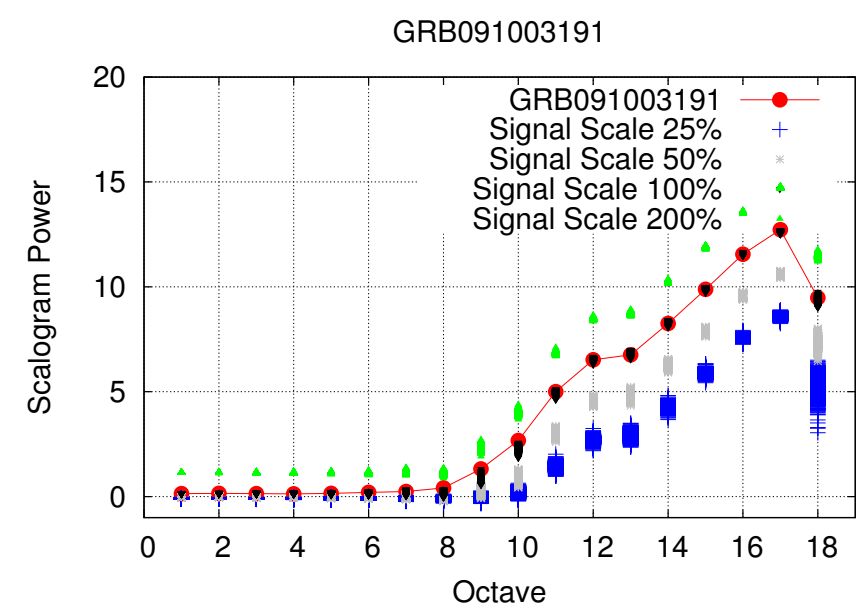

Figure 4: Scalogram for simulated lightcurves with given signal scale factors: RED (Observed lightcurve), BLACK, GREY, BLUE, and GREEN (denoised lightcurves with scale factors: 100\%, 50\%, 25\%, 200\% respectively).

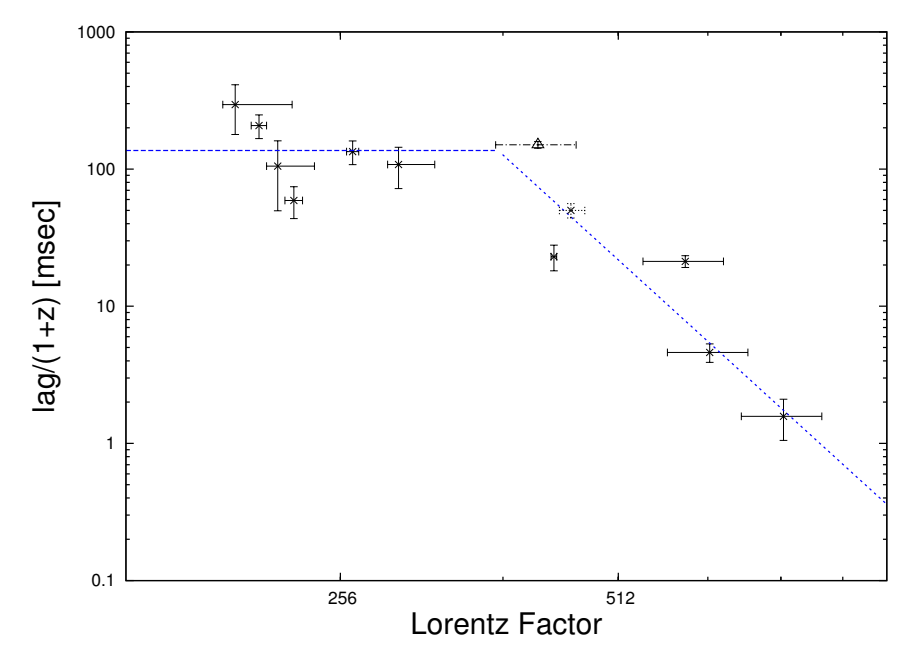

Figure 5: Spectral Lag versus the Lorentz factor.

- The MTS-Liso correlation exhibits the same features as the MTS- $\Gamma$ correlation.

- Possible effects of observational bias in the MTS extraction were estimated via a simulation but the correlations appear to survive.

- The spectral lag vs. $\Gamma$ shows the same features as MTS- $\Gamma$. This correlation should be less sensitive to observational bias.

- We observe an interesting connection between MTS and $t_{p}$, the peak times of the afterglow emission. 


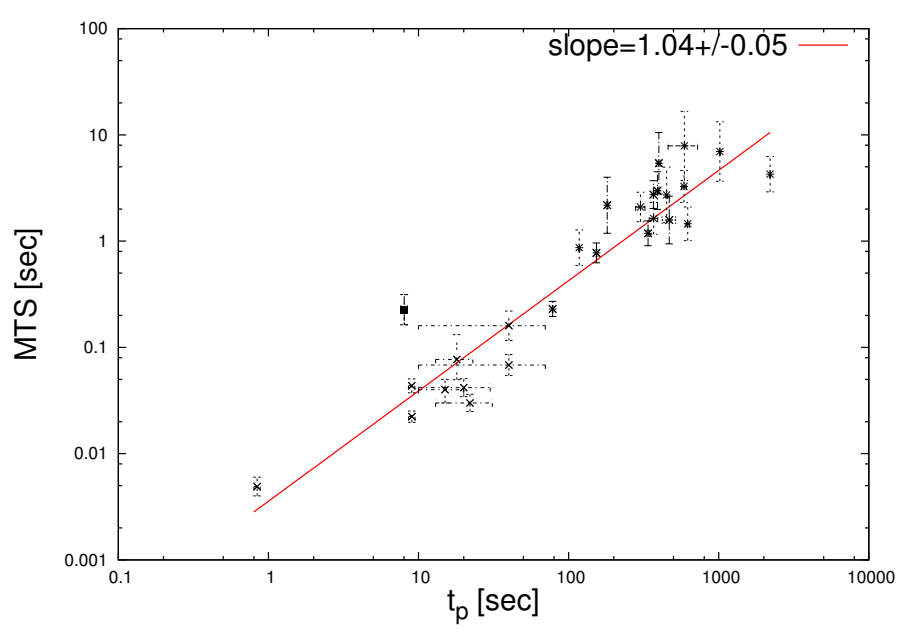

Figure 6: MTS vs. Peak time of the afterglow emission.

These correlations, if substantiated by more precise data (and barring significant observational bias in the extraction of the MTS), can serve as predictors of unknown Lorentz factors for GRBs.

\section{References}

[1] Donoho, D. L., Statistical Estimation and Optimal Recovery, Ann. Stat., 1994, 22238.

[2] Ghirlanda, G. Nava, L.; Ghisellini, G. et al. Gamma-ray bursts in the comoving frame, MNRAS, 2012, 420483.

[3] Ghisellini, G. Ghirlanda, G., Nava, L., Celotti, A., GeV emission from gamma-ray bursts: a radiative fireball?, MNRAS, 2010, 403, 926

[4] Kumar, P. \& Duran, B. R. External forward shock origin of high-energy emission for three gamma-ray bursts detected by Fermi, MNRAS, 2010, 409, 226

[5] Lu, J. Zou, Y.-C., Lei, W.-H. et al. Lorentz-factor-Isotropic-luminosity/Energy Correlations of Gamma-Ray Bursts and Their Interpretation, ApJ, 2012, 751, 49

[6] MacLachlan, G. A., Shenoy, A., Sonbas, E. et al. Minimum variability time-scales of long and short GRBs, MNRAS, 2013, 432, 857 